\title{
Erratum to: Compared antioxidant activity among corticosteroids on cultured retinal pigment epithelial cells
}

\author{
Raffaele Nuzzi $^{1}$ - Alessandro Marchese ${ }^{1}$ - Dario Ghigo ${ }^{2}$
}

Published online: 7 November 2016

(C) Springer-Verlag Berlin Heidelberg 2016

Erratum to: Graefes Arch Clin Exp Ophthalmol

DOI 10.1007/s00417-016-3519-3

Reason: Incorrect order of first name and last name - i.e. last name abbreviated instead of the first name (E.g. N. Raffaele, instead of R. Nuzzi).

Corrected version: Raffaele Nuzzi, Alessandro Marchese, Dario Ghigo

The online version of the original article can be found at http://dx.doi. org/10.1007/s00417-016-3519-3.

Alessandro Marchese amarchese@hotmail.it

1 Division of Ophthalmology, Department of Surgical Sciences, University of Turin, San Luigi Gonzaga Hospital, Regione Gonzole, 10, 10043 Orbassano, TO, Italy

2 Department of Oncology, University of Turin, Turin, Italy 